\title{
Fundamental collapse of the exciton-exciton effective scattering
}

\author{
Laura Pilozzi ${ }^{1}$, Monique Combescot ${ }^{2}$, Odile Betbeder-Matibet ${ }^{2}$, Andrea D'Andrea ${ }^{1}$ \\ (1) Istituto dei Sistemi Complessi, CNR, C.P. 10, Monterotondo Stazione, Roma I-00016 and \\ (2) Institut des NanoSciences de Paris, CNRS, Université Pierre et Marie Curie, 140 rue de Lourmel, 75015 Paris
}

(Dated: November 28, 2018)

\begin{abstract}
The exciton-exciton effective scattering which rules the time evolution of two excitons is studied as a function of initial momentum difference, scattering angle and electron-to-hole mass ratio. We show that this effective scattering can collapse for energy-conserving configurations provided that the difference between the two initial exciton momenta is larger than a threshold value. Sizeable scatterings then exist in the forward direction only. We even find that, for an electron-to-hole mass ratio close to $1 / 2$, the exciton-exciton effective scattering stays close to zero in all directions when the difference between the initial exciton momenta has a very specific value. This unexpected but quite remarkable collapse comes from tricky compensation between direct and exchange Coulomb processes which originates from the fundamental undistinguishability of the exciton fermionic components.
\end{abstract}

PACS numbers: 71.35.-y

\section{INTRODUCTION}

It is now commonly accepted that the composite boson nature of excitons plays a key role in their many-body physics: The undistinguishability of the two carriers from which excitons are constructed leads to all kinds of elaborate exchange processes which can hardly be handled within a naïve bosonic framework.

These exchange processes enter exciton-exciton interactions which are known to be the main source of nonlinearity in optical properties of semiconductors. Among them, we can cite stimulated scattering ${ }^{1}$, polarization change $^{2}$, bistable ${ }^{3}$ and multistable ${ }^{4}$ behaviors and exciton spin relaxation ${ }^{5}$.

Exchange between indistinguishable quantum particles has also been shown to play a fundamental role in the physics of Bose-Einstein condensation. ${ }^{6}$ Interest of this argument is renewed by recent observations of polariton condensates ${ }^{7,8}$, polaritons being mixed states of one exciton and one photon. In pioneering experiments ${ }^{9}$ addressed to fluid propagation of a coherent polariton gas $^{10,11}$, the formation of vortices ${ }^{12}$ has just been evidenced. It is however worth noting that, although theoretically predicted much longer ago ${ }^{13,14}$, the experimental observation of Bose-Einstein condensation of a pure exciton gas remains a challenge ${ }^{15,16}$, in spite of very many different attempts ${ }^{17-21}$. Possible reason for not observing exciton Bose-Einstein condensation can actually be due to the fact that excitons must condense into a dark state $^{22}$. Here too, carrier exchanges play a key role since exchange between two opposite spin bright excitons produce two opposite spin dark excitons.

A correct handling of the exciton composite nature thus is a request, not only from the theoretical point of view, but also to correctly understand the experimental data.

One of the most drastic mathematical difference between elementary bosons and composite bosons made of two free fermions with momenta $\left(\mathbf{k}_{e}, \mathbf{k}_{h}\right)$, as the Wannier excitons, is the fact that the prefactor in the $N$-particle closure relation is $(1 / N !)^{2}$ when the particle composite nature is kept while it is $(1 / N$ !) only when these particles are replaced by elementary bosons ${ }^{23,24}$. This prefactor difference proves that the formal replacement of Wannier excitons by elementary bosons is a dream, even in the extreme dilute limit of just $N=2$ excitons, because all sum rules which result from closure relation, are going to be different, whatever the exciton-exciton effective scatterings.

By contrast, it is worth noting that the closure relation for Frenkel excitons ${ }^{24-27}$, which are made of electron-hole pairs localized on the same ion site, has the same $(1 / N$ !) prefactor as the one of elementary bosons. The reason is that, instead of two degrees of freedom $\left(\mathbf{k}_{e}, \mathbf{k}_{h}\right)$, Frenkel excitons have one only: the ion site $n$.

From a mathematical point of view, the composite nature of particles constructed on two free fermions makes the exciton basis for $N$-pair states overcomplete - except for $N=1$. As a mere consequence, the $N$ Wannier-exciton states are not orthogonal. While a nonorthogonal basis is rather easy to handle, the intrinsic overcompleteness of the Wannier-exciton state basis cannot be eliminated in a self-consistent way. This makes all attempts ${ }^{28,29}$ to work with an orthogonalized exciton state set, doomed to failure because the difficulty is not so much to find a procedure to orthogonalize the states but to reduce their number consistently. In the following, we will restrict to Wannier excitons since those are the ones for which the composite-boson nature shows up the most dramatically.

Having, on the one hand, understood the intrinsic difficulty linked to the overcompleteness of $N$-pair states when written in terms of exciton operators, being, on the other hand, fully convinced that these exciton states constitute the relevant basis ${ }^{30-32}$ for a proper description of many-body effects in a dilute system of electron-hole pairs, we have recently constructed a formalism ${ }^{33}$ which allows us to handle this overcompleteness in an exact way. 


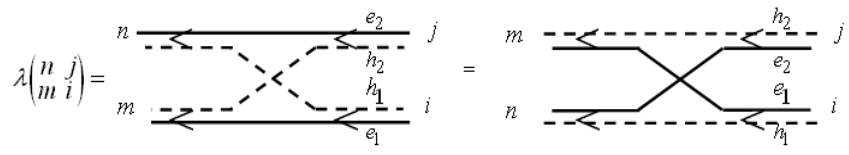

FIG. 1: Pauli scattering $\lambda\left(\begin{array}{ll}n & j \\ m & i\end{array}\right)$ for fermion exchange between two excitons starting in "in" states $(i, j)$ and ending in "out" states $(m, n)$. Electrons are represented by solid lines and holes by dashed lines.

The conceptual difference between our formalism and the Green function formalism developed long ago for elementary quantum particles lies in the fact that this composite boson formalism uses an operator algebra based on commutators between exciton creation operators ${ }^{34}$, while the Green function formalism relies on scalars only.

The link between the overcompleteness of exciton states and the exciton composite nature is evidenced through the relation

$$
B_{i}^{\dagger} B_{j}^{\dagger}=-\sum_{m n} \lambda\left(\begin{array}{ll}
n & j \\
m & i
\end{array}\right) B_{m}^{\dagger} B_{n}^{\dagger}
$$

which comes from the two different ways to associate two electrons and two holes into two Wannier excitons. $B_{i}^{\dagger}$ is the creation operator for exciton $i$ having $\mathbf{K}_{i}$ as center-ofmass momentum and $\nu_{i}$ as relative motion index. The 2 by 2 Pauli scattering $\lambda\left(\begin{array}{ll}n & j \\ m & i\end{array}\right)$, shown in Fig.1, describes fermion exchange between two excitons in states $(i, j)$ in the absence of fermion interaction. The real difficulty with composite excitons is to produce a formalism unchanged with respect to the above identity. Our composite-boson many-body theory ${ }^{33}$ does it in an exact self-consistent way.

As $\lambda\left(\begin{array}{ll}n & j \\ m & i\end{array}\right)$ in Eq.(1) is a dimensionless quantity, such pure exchange scatterings cannot appear alone in effective scatterings ruling the time evolution of two excitons because these effective scatterings must be energy-like quantities. However, when mixed with Coulomb process, these carrier exchanges, which come from the intrinsic undistinguishablity of the exciton fermionic components, become crucial because they readily lead to six different energy-like scatterings between two excitons. These depend on how the carriers of the two excitons are associated in the "in" and "out" states $(i, j)$ and $(m, n)$. The resulting six different scatterings are shown in Figs. $(2,3)$. In the direct Coulomb scattering $\xi^{\operatorname{dir}}\left(\begin{array}{ll}n & j \\ m & i\end{array}\right)$, shown in Fig.2(a), the excitons $m$ and $i$ are made with the same carriers while in $\xi^{\operatorname{dir}}\left(\begin{array}{cc}m & j \\ n & i\end{array}\right)$ obtained by a $(m, n)$ permutation, their two carriers are different, so that $\xi^{\operatorname{dir}}\left(\begin{array}{cc}m & j \\ n & i\end{array}\right)$ can be seen as a direct Coulomb scattering followed by two Pauli scatterings for carrier exchange in the absence of carrier interaction [see Fig.2(b)]. The direct Coulomb

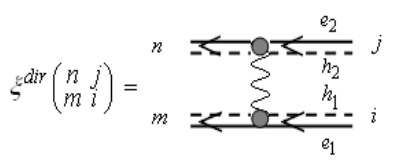

(a)

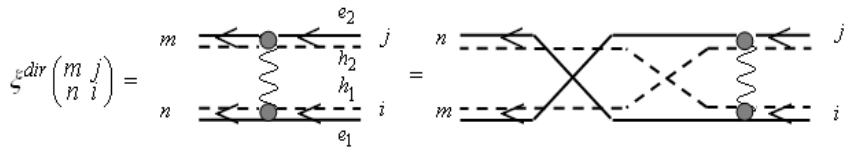

(b)

FIG. 2: (a): Direct interaction scatterings between two excitons starting in "in" states $(i, j)$ and ending in "out" states $(m, n)$ for carrier interaction in the absence of carrier exchange. (b): A $(m, n)$ permutation can be seen as the result of a double carrier exchange.

scattering $\xi^{\operatorname{dir}}\left(\begin{array}{ll}n & j \\ m & i\end{array}\right)$ is given by ${ }^{33}$

$$
\begin{aligned}
\xi^{\operatorname{dir}} & \left(\begin{array}{ll}
n & j \\
m & i
\end{array}\right)=\int\{d \mathbf{r}\}\left(V_{e_{1} e_{2}}+V_{h_{1} h_{2}}-V_{e_{1} h_{2}}-V_{e_{2} h_{1}}\right) \\
& \times\left\langle m \mid \mathbf{r}_{e_{1}}, \mathbf{r}_{h_{1}}\right\rangle\left\langle n \mid \mathbf{r}_{e_{2}}, \mathbf{r}_{h_{2}}\right\rangle\left\langle\mathbf{r}_{h_{2}}, \mathbf{r}_{e_{2}} \mid j\right\rangle\left\langle\mathbf{r}_{h_{1}}, \mathbf{r}_{e_{1}} \mid i\right\rangle
\end{aligned}
$$

$\left\langle\mathbf{r}_{h_{1}}, \mathbf{r}_{e_{1}} \mid i\right\rangle$ is the wave function of an exciton in state $i$, its electron being located at $\mathbf{r}_{e_{1}}$ and its hole at $\mathbf{r}_{h_{1}}$. Coulomb interaction between two electrons reads $V_{e_{1} e_{2}}=$ $e^{2} /\left|\mathbf{r}_{e_{1}}-\mathbf{r}_{e_{2}}\right|$ and similarly for the other Coulomb terms. The above expression of $\xi^{\operatorname{dir}}\left(\begin{array}{ll}n & j \\ m & i\end{array}\right)$ visually follows from the diagrammatic representation of Fig.2(a).

In addition to direct Coulomb scatterings, two excitons can also have exchange Coulomb scatterings. In $\xi^{\text {in }}\left(\begin{array}{ll}n & j \\ m & i\end{array}\right)$ and $\xi^{\text {out }}\left(\begin{array}{ll}n & j \\ m & i\end{array}\right)$, the excitons $m$ and $i$ have the same electron but a different hole, while in $\xi^{\text {in }}\left(\begin{array}{cc}m & j \\ n & i\end{array}\right)$ and $\xi^{\text {out }}\left(\begin{array}{ll}m & j \\ n & i\end{array}\right)$ obtained from a $(m, n)$ permutation, they have the same hole but a different electron. These exchange Coulomb scatterings result from a succession of direct Coulomb scattering and Pauli scattering for carrier exchange. Fig.3(a) shows that $\xi^{\text {in }}\left(\begin{array}{ll}n & j \\ m & i\end{array}\right)$ reads as $\xi^{\text {dir }}\left(\begin{array}{ll}n & j \\ m & i\end{array}\right)$ with $\left(\mathbf{r}_{h_{1}}, \mathbf{r}_{h_{2}}\right)$ exchanged in the $(m, n)$ wave functions.

It turns out that, as briefly rederived below, $\xi^{\text {out }}$ in which Coulomb interaction takes place after carrier exchange, does not enter the effective scattering ruling the time evolution of two excitons ${ }^{35,36}$. This is due to a very fundamental reason linked to symmetry breaking in the evolution towards positive time. It however is of interest to note that, as physically expected, a symmetry between these two exchange Coulomb scatterings exists in the large time limit: indeed, we do have ${ }^{33}$

$\xi^{\text {in }}\left(\begin{array}{ll}n & j \\ m & i\end{array}\right)-\xi^{\text {out }}\left(\begin{array}{ll}n & j \\ m & i\end{array}\right)=\left(E_{m}+E_{n}-E_{i}-E_{j}\right) \lambda\left(\begin{array}{ll}n & j \\ m & i\end{array}\right)$.

where $E_{i}$ is the $i$ exciton energy, so that the two exchange 


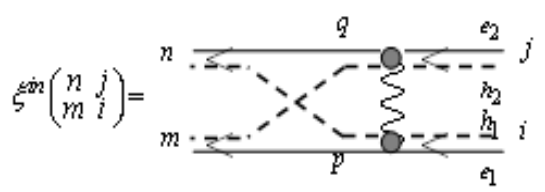

(a)

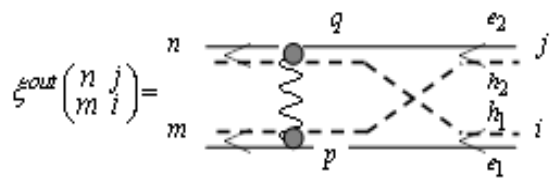

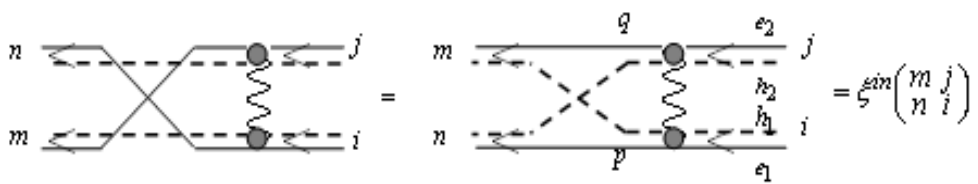

(b)

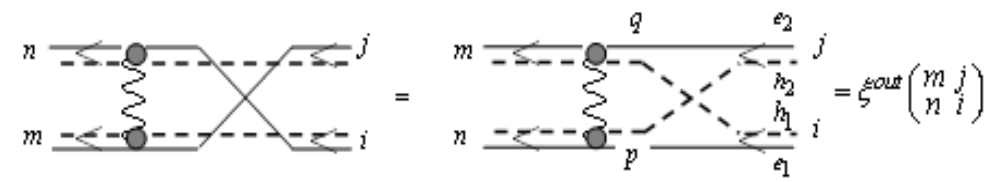

FIG. 3: Coulomb exchange scatterings: in $\xi^{\text {in }}$, the Coulomb processes take place between the "in" excitons, while in $\xi^{\text {out }}$ they take place between the "out" excitons. These exchange Coulomb scatterings can be seen as a succession of a direct Coulomb scattering and a Pauli scattering for carrier exchange in the absence of carrier interaction.

Coulomb scatterings are equal for energy-conserving processes, i.e., when time reversal is expected.

Even if $\xi^{\text {out }}$ does not enter the effective scattering ruling the time evolution of two excitons, we are nevertheless left with four different energy-like quantities. Being equally relevant since they only differ by the intrinsic fermion undistinguishability, these four scatterings must appear on equal footing in the effective scattering ruling the time evolution of two excitons. Consequently, this effective scattering must read as a linear combination of four terms, each of these four terms containing two Coulomb attractions and two Coulomb repulsions. Such a complex structure is a direct consequence of the particle composite nature. Some tricky compensations can then take place in this linear combination, to possibly end with an effective scattering, either very close to zero, or even exactly equal to zero for some specific configurations; the corresponding initial state can then be seen as "frozen" at first order in Coulomb processes.

In a previous work ${ }^{36}$, we found that the effective scattering of two excitons having same initial momentum cancels for a finite value of the momentum transfer. This particular transfer however has no physical relevance because it does not correspond to process in which energy is conserved. Being still puzzled by this somewhat unexpected cancellation, we wanted to reconsider the problem more in details in order to see if the exciton-exciton effective scattering which rules the time evolution of two excitons can cancel for some energy-conserving configurations. This is the purpose of the present work.

We here show that, indeed, there are some configurations in which the effective scattering ruling the time evolution of two excitons does cancel while energy is conserved. As a result, the corresponding scattering configurations are forbidden at first order in the interaction. For some electron-to-hole mass ratio close to $1 / 2$, the effective exciton-exciton scattering can even stay very close to zero in all directions provided that the initial momentum difference has a very specific value. This particular initial configuration then appears as somewhat magic because excitons do not scatter through first-order Coulomb process. Such a cancellation however requires initial exciton momenta above a threshold value which is far larger than the typical photon momenta, i.e., the momenta of the photocreated excitons. Excitons in their relative motion ground state with a larger kinetic energy can however be formed through collisions between excited state excitons resulting from photon excitation above the absorption edge. However, independently from its possible observation, it is of importance to understand that the excitonexciton effective scattering can collapse as a result of the exciton composite nature. To reveal its existence thus constitutes a relevant part of the overall understanding of exciton-exciton interaction.

The present paper is organized as follows.

In section II, we construct the effective scattering which rules the time evolution of two excitons as imposed by the particle quantum nature. In order to better grasp the importance of the fermion/boson nature of the particles as well as the consequence of fermionic components in this effective scattering, we here briefly rederive the time evolution of two elementary fermions, two elementary bosons, and two Wannier excitons. This allows us to evidence that a possible collapse of the effective scattering is a fundamental property of elementary fermions, this collapse appearing above a threshold only in the case of composite bosons made of two fermions.

In section III, we study the possible cancellation of this effective exciton-exciton scattering for energy conserving configurations. To this end, we perform a numerical calculation of the "in" exchange Coulomb scattering appearing in this effective scattering in the most general case, i.e., for initial excitons having different momenta and arbitrary mass ratio. We then restrict to energy-conserving configurations and study the dependence of the effective scattering on exciton momentum difference, scattering 


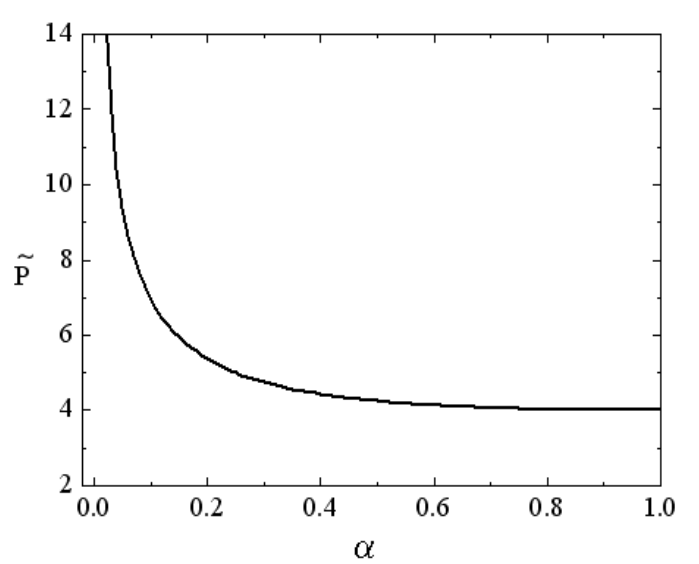

FIG. 4: Exciton momenta in 3D Bohr radius unit $\tilde{P}=a_{X} P$, for which the exciton kinetic energy is equal to its binding energy, as a function of mass ratio $\alpha=\frac{m_{e}}{m_{h}}$.

angle and electron-to-hole mass ratio. We pay particular attention to the magic configuration in which the effective scattering stays close to zero in all directions.

In section IV, we conclude.

\section{EFFECTIVE SCATTERING FOR THE TIME EVOLUTION OF TWO QUANTUM PARTICLES}

\section{A. Relevant coordinates}

In order to analyse the exciton momentum configuration possibly leading to a cancellation of the exciton effective scattering, we will, for simplicity, restrict to $2 \mathrm{D}$ scatterings in which the excitons stay in their relative motion ground state, i.e., processes in which all the relative motion indices $\nu$ are equal to $\nu_{0}$. This restriction requires two conditions to be met:

1) The quantum well should be narrow enough to possibly consider one confined level only.

2) The exciton momenta, P, should be small enough to avoid scattering towards unbound electron-hole pairs. This essentially imposes an exciton kinetic energy smaller than the $2 \mathrm{D}$ binding energy, namely, $P^{2} / 2 M \leq 4 / 2 \mu a_{X}^{2}$, where $M=m_{e}+m_{h}$ is the exciton center-of-mass mass, $\mu^{-1}=m_{e}^{-1}+m_{h}^{-1}$ the inverse exciton relative motion mass and $a_{X}$ the $3 \mathrm{D}$ exciton Bohr radius. For $\tilde{P}=a_{X} P$, this condition reads $\tilde{P} \leq 2(1+\alpha) / \sqrt{\alpha}$. It is shown in Fig. 4 as a function of the mass ratio $\alpha=m_{e} / m_{h}$.

Also for simplicity, we will not here consider the exciton spin degrees of freedom: this physically corresponds to take the electrons (holes) of the two excitons with same spin.

Since the total center-of-mass momentum $2 \mathbf{K}$ of two excitons is conserved in a scattering process, we are led to write the center-of-mass momenta of the two initial excitons as $\mathbf{K}_{i}=\mathbf{K}+\mathbf{P}$ and $\mathbf{K}_{j}=\mathbf{K}-\mathbf{P}$, while the ones
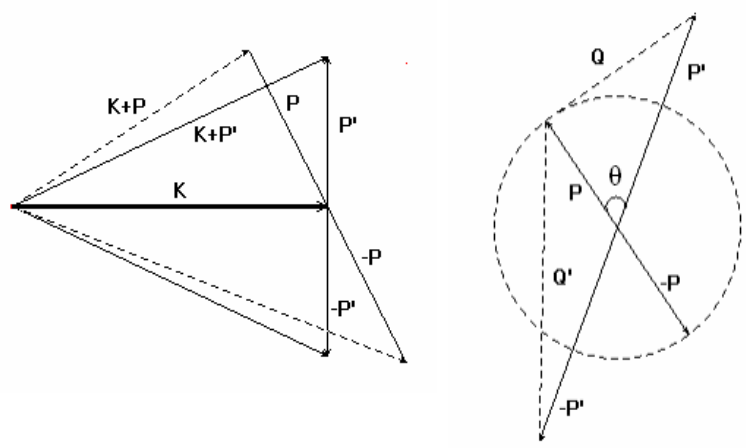

FIG. 5: (a): In the laboratory frame, the "in" excitons with momenta $\mathbf{K}_{i}=\mathbf{K}+\mathbf{P}$ and $\mathbf{K}_{j}=\mathbf{K}-\mathbf{P}$ transform into "out" excitons with momenta $\mathbf{K}_{m}=\mathbf{K}+\mathbf{P}^{\prime}$ and $\mathbf{K}_{n}=\mathbf{K}-\mathbf{P}^{\prime}$. (b): In the center-of-mass frame, which corresponds to set $\mathbf{K}=0$, these excitons have momenta $(\mathbf{P},-\mathbf{P})$ and $\left(\mathbf{P}^{\prime},-\mathbf{P}^{\prime}\right)$. Energy conserving processes, which are the relevant ones in the large time limit, lead to $P=P^{\prime}$ : momenta follow a simple rotation in the center-of-mass frame, as shown by the dashed circle.

of the two final excitons are written as $\mathbf{K}_{m}=\mathbf{K}+\mathbf{P}^{\prime}$ and $\mathbf{K}_{n}=\mathbf{K}-\mathbf{P}^{\prime}$ [see Fig.5(a)]. As physical results cannot depend on frame momentum, we can, without any loss of generality, set $\mathbf{K}$ equal to zero. This leads us to rewrite the effective scattering for the configuration of interest as, [see Fig.6],

$$
\xi^{\mathrm{eff}}\left(\begin{array}{ll}
n & j \\
m & i
\end{array}\right) \equiv \xi^{\mathrm{eff}}\left(\begin{array}{cc}
-\mathbf{P}^{\prime} & -\mathbf{P} \\
\mathbf{P}^{\prime} & \mathbf{P}
\end{array}\right) .
$$

It will also appear as convenient to introduce the two momentum transfers $\left(\mathbf{Q}, \mathbf{Q}^{\prime}\right)$ of this scattering process. These are defined as

$$
\mathbf{P}+\mathbf{Q}=\mathbf{P}^{\prime} \quad \mathbf{P}+\mathbf{Q}^{\prime}=-\mathbf{P}^{\prime}:
$$

due to carrier undistinguishability, exciton with initial momentum $\mathbf{P}$ can as well end with the final momentum $\mathbf{P}^{\prime}$ or $-\mathbf{P}^{\prime}$, which corresponds to change $\mathbf{Q}$ into $\mathbf{Q}^{\prime}$ [see Fig.5(b)].

Since $\mathbf{Q}=\mathbf{P}^{\prime}-\mathbf{P}$, while $\mathbf{Q}^{\prime}=-\mathbf{P}^{\prime}-\mathbf{P}$, these momentum transfers are such that $Q=Q^{\prime}$ for $\mathbf{P} . \mathbf{P}^{\prime}=0$. We can also note that, for scatterings staying within the same exciton relative motion subspace, i.e., for excitons all having the same relative motion index $\nu_{0}$, energy conservation imposes $(\mathbf{K}+\mathbf{P})^{2}+(\mathbf{K}-\mathbf{P})^{2}=\left(\mathbf{K}+\mathbf{P}^{\prime}\right)^{2}+\left(\mathbf{K}-\mathbf{P}^{\prime}\right)^{2}$, i.e., $P=P^{\prime}$ : the scattered momentum then evolves on a radius $P$ circle. This $P=P^{\prime}$ condition also reads $\mathbf{Q} \cdot \mathbf{Q}^{\prime}=0$. As $\mathbf{Q}+\mathbf{Q}^{\prime}=-2 \mathbf{P}$, the condition $\mathbf{Q} \cdot \mathbf{Q}^{\prime}=0$

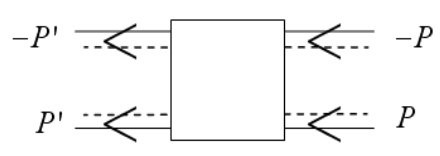

FIG. 6: Scattering of two excitons with initial momenta $(\mathbf{P},-\mathbf{P})$ and final momenta $\left(\mathbf{P}^{\prime},-\mathbf{P}^{\prime}\right)$ in the center-of-mass frame. 
implies $Q^{2}+Q^{\prime 2}=4 P^{2}$. This shows that, for a given $P$, the final states having the energy of the initial state, are fully determined by the scattering angle $\theta$ between $\mathbf{P}$ and $\mathbf{P}^{\prime}$.

As we are mainly interested in energy conserving processes, we will ultimately study the effective scattering $\xi^{\text {eff }}\left(\begin{array}{rr}-\mathbf{P}^{\prime} & -\mathbf{P} \\ \mathbf{P}^{\prime} & \mathbf{P}\end{array}\right)$ of two excitons as a function of the scattering angle $\theta$ and half the initial exciton momentum difference $P=\left|\mathbf{K}_{i}-\mathbf{K}_{j}\right| / 2$, for various electron-to-hole mass ratios.

\section{B. Effective scattering for elementary particles}

To better grasp the importance of the particle composite nature and to relate the possible collapse of the effective $2 \times 2$ scattering to the particle fermionic/bosonic nature, let us first consider two elementary quantum particles having initial momenta $(\mathbf{P},-\mathbf{P})$ and final momenta $\left(\mathbf{P}^{\prime},-\mathbf{P}^{\prime}\right)$ in the center-of-mass frame $(\mathbf{K}=\mathbf{0})$. The time evolution of the initial state $(\mathbf{P},-\mathbf{P})$ is given by

$$
\left|\psi_{t}\right\rangle=e^{-i H t} C_{\mathbf{P}}^{\dagger} C_{-\mathbf{P}}^{\dagger}|v\rangle
$$

where $C_{\mathbf{P}}^{\dagger}$ creates the elementary particle of interest with momentum $\mathbf{P}$. Depending on the particle quantum nature, these operators are such that

$$
C_{\mathbf{P}_{1}} C_{\mathbf{P}_{2}}^{\dagger}+\eta C_{\mathbf{P}_{2}}^{\dagger} C_{\mathbf{P}_{1}}=\delta_{\mathbf{P}_{2}, \mathbf{P}_{1}},
$$

with $\eta=1$ for fermions and $\eta=-1$ for bosons. The above equation leads to $\left\langle v\left|C_{-\mathbf{P}^{\prime}} C_{\mathbf{P}^{\prime}} C_{\mathbf{P}}^{\dagger} C_{-\mathbf{P}}^{\dagger}\right| v\right\rangle=\delta_{\mathbf{P}^{\prime}, \mathbf{P}}+$ $\eta \delta_{\mathbf{P}^{\prime},-\mathbf{P}}$.

The simplest way to calculate $\left|\psi_{t}\right\rangle$ is to use the integral representation of the exponential. For $t>0$, it reads

$$
e^{-i H t}=\int_{-\infty}^{+\infty} \frac{d x}{(-2 i \pi)} \frac{e^{-i t\left(x+i O_{+}\right)}}{x+i O_{+}-H},
$$

where $O_{+}$is an arbitrary positive constant. The probability to go from $(\mathbf{P},-\mathbf{P})$ to $\left(\mathbf{P}^{\prime},-\mathbf{P}^{\prime}\right)$ is thus given by

$$
\begin{gathered}
\left\langle v\left|C_{-\mathbf{P}^{\prime}} C_{\mathbf{P}^{\prime}} e^{-i H t} C_{\mathbf{P}}^{\dagger} C_{-\mathbf{P}}^{\dagger}\right| v\right\rangle=\int_{-\infty}^{+\infty} \frac{d x}{(-2 i \pi)} e^{-i t\left(x+i O_{+}\right)} \\
\times\left\langle v\left|C_{-\mathbf{P}^{\prime}} C_{\mathbf{P}^{\prime}} \frac{1}{x+i O_{+}-H} C_{\mathbf{P}}^{\dagger} C_{-\mathbf{P}}^{\dagger}\right| v\right\rangle
\end{gathered}
$$

To go further and calculate the matrix element in this integral, we use the following identity valid for $H=H_{0}+$ $V$, this identity being the key for correlation effects with elementary quantum particles,

$$
\frac{1}{z-H}=\frac{1}{z-H_{0}}+\frac{1}{z-H} V \frac{1}{z-H_{0}} .
$$

For $H_{0}=\sum_{\mathbf{k}} \epsilon_{\mathbf{k}} C_{\mathbf{k}}^{\dagger} C_{\mathbf{k}}$ and $V$ given by

$$
V=\frac{1}{2} \sum_{\mathbf{q} \neq \mathbf{0}} V_{\mathbf{q}} \sum_{\mathbf{k}_{1}, \mathbf{k}_{2}} C_{\mathbf{k}_{1}+\mathbf{q}}^{\dagger} C_{\mathbf{k}_{2}-\mathbf{q}}^{\dagger} C_{\mathbf{k}_{2}} C_{\mathbf{k}_{1}},
$$

this leads us to write, at first order in the interaction,

$$
\begin{aligned}
& \left\langle v\left|C_{-\mathbf{P}^{\prime}} C_{\mathbf{P}^{\prime}} e^{-i H t} C_{\mathbf{P}}^{\dagger} C_{-\mathbf{P}}^{\dagger}\right| v\right\rangle \simeq \\
& \quad e^{-2 i \epsilon_{\mathbf{P}} t}\left(\delta_{\mathbf{P}, \mathbf{P}^{\prime}}+\eta \delta_{\mathbf{P},-\mathbf{P}^{\prime}}\right) \\
& \quad+\left(V_{\mathbf{P}^{\prime}-\mathbf{P}}+\eta V_{\mathbf{P}^{\prime}+\mathbf{P}}\right) \frac{e^{-2 i \epsilon_{\mathbf{P}^{\prime}} t}-e^{-2 i \epsilon_{\mathbf{P}} t}}{2 \epsilon_{\mathbf{P}^{\prime}}-2 \epsilon_{\mathbf{P}}}+\cdots
\end{aligned}
$$

This shows that, due to the quantum particle undistinguishability, the effective scattering which rules the time evolution of two elementary particles starting in state $(\mathbf{P},-\mathbf{P})$ and ending in a different state $\left(\mathbf{P}^{\prime},-\mathbf{P}^{\prime}\right)$ is made of two processes which differ by a particle exchange,

$$
\xi^{\mathrm{eff}}\left(\begin{array}{rr}
-\mathbf{P}^{\prime} & -\mathbf{P} \\
\mathbf{P}^{\prime} & \mathbf{P}
\end{array}\right)=V_{\mathbf{P}^{\prime}-\mathbf{P}}+\eta V_{\mathbf{P}^{\prime}+\mathbf{P}}=V_{\mathbf{Q}}+\eta V_{\mathbf{Q}^{\prime}} .
$$

In the case of two fermions, this effective scattering reduces to $V_{\mathbf{Q}}-V_{\mathbf{Q}^{\prime}}$, the minus sign being standard for process associated to fermion exchange. This shows that the effective scattering ruling the time evolution of two elementary fermions cancels for $Q=Q^{\prime}$, i.e., for $\mathbf{P}^{\prime}$ perpendicular to $\mathbf{P}$, with in addition $P^{\prime}=P$ in the case of energy-conserving processes. Let us stress that this effective scattering sign change should not be taken as a change from repulsion to attraction between the particles at hand: this latter characteristic is fully linked to the sign of the elementary scattering $V_{\mathbf{Q}}$ in the Hamiltonian, not to the sign of the effective scattering ruling the time evolution of two fermions, as obvious from the fact that this effective scattering anyway appears as a square modulus in the Fermi golden rule.

By contrast, the effective scattering of two elementary bosons is given by $V_{\mathbf{Q}}+V_{\mathbf{Q}^{\prime}}$, so that such a cancellation does not occur. The possible cancellation of the effective scattering ruling the time evolution of two elementary quantum particles thus appears as a characteristic of the particle fermionic nature. A memory of this fermionic cancellation is going to show up in the case of excitons made of two free fermions, but above a momentum threshold only.

\section{Effective scattering for two-fermion particles}

We now turn to the time evolution of two Wannier excitons made of linear combination of free fermion pairs. Let $B_{i}^{\dagger}$ be the creation operator of one exciton in state $i$. This operator is such that $\left(H-E_{i}\right) B_{i}^{\dagger}|v\rangle=0$. Due to the exciton composite nature, the scalar product of two-exciton states is given by ${ }^{33}$

$$
\left\langle\phi_{m n} \mid \phi_{i j}\right\rangle=\left[\delta_{m, i} \delta_{n, j}-\lambda\left(\begin{array}{ll}
n & j \\
m & i
\end{array}\right)\right]+[m \leftrightarrow n],
$$

where $\left|\phi_{i j}\right\rangle=B_{i}^{\dagger} B_{j}^{\dagger}|v\rangle$ and $\lambda\left(\begin{array}{ll}n & j \\ m & i\end{array}\right)$ is the Pauli scattering for fermion exchange in the absence of fermion interaction, shown in Fig.1.

To get the time evolution of the two-exciton state $B_{i}^{\dagger} B_{j}^{\dagger}|v\rangle$, we use a procedure similar to the one we have 
used in the case of elementary quantum particles. Equation (8) allows us to write

$$
\begin{aligned}
\left\langle\phi_{m n}\left|e^{-i H t}\right| \phi_{i j}\right\rangle & =\int_{-\infty}^{+\infty} \frac{d x}{(-2 i \pi)} e^{-i t\left(x+i O_{+}\right)} \\
& \times\left\langle\phi_{m n}\left|\frac{1}{x+i O_{+}-H}\right| \phi_{i j}\right\rangle .
\end{aligned}
$$

Since the semiconductor Hamiltonian does not split in terms of exciton operators as $H_{\mathrm{X}}+V_{\mathrm{Xx}}$, we cannot use Eq.(10). Correlations betwen excitons then follow from a similar equation in which enters the exciton creation operator, namely ${ }^{33}$,

$$
\frac{1}{z-H} B_{i}^{\dagger}=B_{i}^{\dagger} \frac{1}{z-H-E_{i}}+\frac{1}{z-H} V_{i}^{\dagger} \frac{1}{z-H-E_{i}},
$$

where the operator $V_{i}^{\dagger}=\left[H, B_{i}^{\dagger}\right]-E_{i} B_{i}^{\dagger}$ describes the interactions of exciton $i$ with the rest of the system. To go further, we introduce the direct Coulomb scattering $\xi^{\operatorname{dir}}\left(\begin{array}{ll}n & j \\ m & i\end{array}\right)$ formally defined $\mathrm{as}^{33}$

$$
\left[V_{i}^{\dagger}, B_{j}^{\dagger}\right]=\sum_{m n} \xi^{\operatorname{dir}}\left(\begin{array}{ll}
n & j \\
m & i
\end{array}\right) B_{m}^{\dagger} B_{n}^{\dagger} .
$$

Its precise value is given in Eq.(2) and its diagrammatic representation is shown in Fig.2(a).

It is then easy to show that, to lowest order in the interaction,

$$
\begin{aligned}
& \left\langle\phi_{m n}\left|\frac{1}{z-H}\right| \phi_{i j}\right\rangle \simeq \frac{1}{z-E_{i j}}\left\langle\phi_{m n} \mid \phi_{i j}\right\rangle \\
& \quad+\frac{1}{\left(z-E_{m n}\right)\left(z-E_{i j}\right)} \sum_{p, q}\left\langle\phi_{m n} \mid \phi_{p q}\right\rangle \xi^{\operatorname{dir}}\left(\begin{array}{ll}
q & j \\
p & i
\end{array}\right)
\end{aligned}
$$

where $E_{i j}=E_{i}+E_{j}$. Using the scalar product of twoexciton states given in Eq.(14), we end with

$$
\begin{aligned}
&\left\langle\phi_{m n}\left|e^{-i H t}\right| \phi_{i j}\right\rangle \simeq \\
& e^{-i E_{i j} t}\left\{\left[\delta_{m, i} \delta_{n, j}-\lambda\left(\begin{array}{cc}
n & j \\
m & i
\end{array}\right)\right]+[m \leftrightarrow n]\right\} \\
&+\frac{e^{-i E_{m n} t}-e^{-i E_{i j} t}}{E_{m n}-E_{i j}} \xi^{\mathrm{eff}}\left(\begin{array}{ll}
n & j \\
m & i
\end{array}\right),
\end{aligned}
$$

where the effective scattering ruling the time evolution of the two excitons $(i, j)$ is given by

$$
\xi^{\mathrm{eff}}\left(\begin{array}{ll}
n & j \\
m & i
\end{array}\right)=\left[\xi^{\mathrm{dir}}\left(\begin{array}{ll}
n & j \\
m & i
\end{array}\right)-\xi^{\mathrm{in}}\left(\begin{array}{ll}
n & j \\
m & i
\end{array}\right)\right]+[m \leftrightarrow n],
$$

with $\xi^{\text {in }}\left(\begin{array}{ll}n & j \\ m & i\end{array}\right)$ being the "in" exchange Coulomb scattering defined as

$$
\xi^{\text {in }}\left(\begin{array}{ll}
n & j \\
m & i
\end{array}\right)=\sum_{p, q} \lambda\left(\begin{array}{ll}
n & q \\
m & p
\end{array}\right) \xi^{\operatorname{dir}}\left(\begin{array}{ll}
q & j \\
p & i
\end{array}\right)
$$

and shown in Fig.3(a).

The undistinguishability of the electron-hole components of the excitons leads to an effective scattering made of four terms instead of two as in the case of elementary quantum particles: starting from the direct Coulomb scattering $\xi^{\operatorname{dir}}\left(\begin{array}{cc}-\mathbf{P}^{\prime} & -\mathbf{P} \\ \mathbf{P}^{\prime} & \mathbf{P}\end{array}\right)$, the three other scatterings correspond either to exchange one electron or one hole as in the two exchange Coulomb scatterings $\xi^{\text {in }}$ of Figs.3 $(a, b)$, or to exchange the two carriers, which is nothing but a $\left(\mathbf{P}^{\prime} \leftrightarrow-\mathbf{P}^{\prime}\right)$ exchange in $\xi^{\text {dir }}$ as shown in Fig.2(b). Since a fermion exchange brings a minus sign, we end with an effective scattering for the process of Fig.6 made of two terms with a plus sign and two terms with a minus sign:

$$
\begin{aligned}
\xi^{\mathrm{eff}}\left(\begin{array}{rr}
-\mathbf{P}^{\prime} & -\mathbf{P} \\
\mathbf{P}^{\prime} & \mathbf{P}
\end{array}\right)=\xi^{\text {dir }}\left(\begin{array}{rr}
-\mathbf{P}^{\prime} & -\mathbf{P} \\
\mathbf{P}^{\prime} & \mathbf{P}
\end{array}\right) & -\xi^{\text {in }}\left(\begin{array}{rr}
-\mathbf{P}^{\prime} & -\mathbf{P} \\
\mathbf{P}^{\prime} & \mathbf{P}
\end{array}\right) \\
& +\left(\mathbf{P}^{\prime} \leftrightarrow-\mathbf{P}^{\prime}\right),
\end{aligned}
$$

each of these four terms being actually made of two Coulomb repulsions and two Coulomb attractions, as seen from Eq.(2). Due to such a complex structure, it is far from obvious to physically guess the sign of the resulting effective scattering and its possible cancellation.

We are going to show that, as for two elementary fermions, the effective scattering for the time evolution of two excitons can cancel. However, for energy conserving process, this cancellation requires a difference between initial momenta larger than a threshold value: for lower initial momentum difference, the exciton bosonic nature dominates: the effective scattering, like for two elementary bosons, keeps a constant sign for all scattering configurations.

Actually, due to a quite subtle interplay between the various Coulomb contributions existing in this effective scattering, interplay which deeply depends on possible symmetry between electron and hole, we even find that, when the electron-to-hole mass ratio is close to $1 / 2$, the effective scattering stays essentially equal to zero in all directions, provided that the initial momentum difference has a very specific value. While the cancellation of the effective scattering for a particular value of the scattering angle is quite standard for elementary fermions, the possible cancellation of this effective scattering in all scattered directions is far more subtle, being deeply linked to the exciton composite nature. It is worth noting that the mass ratio $1 / 2$ to have this somewhat magic cancellation essentially separates hydrogen-like excitons $\left(m_{e} \ll m_{h}\right)$ from positronium-like excitons for which the electron and hole play a quite symmetrical role.

\section{Various contributions to the effective scatterings}

As seen from Eqs.(20) or (22), the effective scattering ruling the time evolution of two excitons is made of two direct terms and two exchange terms. 


\section{Direct terms}

In previous works ${ }^{36,37}$, we showed that the direct exciton-exciton scattering $\xi^{\operatorname{dir}}\left(\begin{array}{rr}-\mathbf{P}^{\prime} & -\mathbf{P} \\ \mathbf{P}^{\prime} & \mathbf{P}\end{array}\right)$ in which excitons with momenta $\mathbf{P}$ and $\mathbf{P}^{\prime}$ are made with the same electron-hole pair, can be written analytically in terms of the exciton momentum transfer $\mathbf{Q}=\mathbf{P}^{\prime}-\mathbf{P}$. In the case of $2 \mathrm{D}$ ground state excitons for which $\left\langle\mathbf{r} \mid \nu_{0}\right\rangle=$ $e^{-2 r / a_{X}} \sqrt{8 / \pi a_{X}^{2}}$, where $a_{X}$ is the $3 \mathrm{D}$ Bohr radius, this scattering reads, in $a_{X}^{-1}$ unit for momentum and $\xi_{X}=$ $e^{2} a_{X} / L^{2}$ unit for scattering,

$$
\begin{aligned}
\xi^{\operatorname{dir}}\left(\begin{array}{rr}
-\mathbf{P}^{\prime} & -\mathbf{P} \\
\mathbf{P}^{\prime} & \mathbf{P}
\end{array}\right) & \equiv \xi_{\alpha_{e}}^{\operatorname{dir}}(Q) \\
& =\frac{2 \pi}{Q}\left[g\left(\alpha_{e} Q\right)-g\left(\alpha_{h} Q\right)\right]^{2}
\end{aligned}
$$

where $\alpha_{e}=1-\alpha_{h}=m_{e} /\left(m_{e}+m_{h}\right)$ while $g(q)=(1+$ $\left.q^{2} / 16\right)^{-3 / 2}$. This shows that $\xi_{\alpha_{e}}^{\operatorname{dir}}(Q)$ reduces to zero for $Q=0$ and $Q$ infinite, while it stays equal to zero for $\alpha_{e}=1 / 2$, i.e., for equal electron and hole masses. Such a cancellation can be physically understood by noting that the exciton composite nature does not show up in a direct scattering, so that excitons basically behave as two classical dipoles, these dipoles being fully symmetrical when the electron and hole masses are equal.

\section{Exchange terms}

By contrast, the "in" exchange scattering cannot be calculated analytically. Its most compact expression appears to read $^{36}$

$$
\xi^{\text {in }}\left(\begin{array}{rr}
-\mathbf{P}^{\prime} & -\mathbf{P} \\
\mathbf{P}^{\prime} & \mathbf{P}
\end{array}\right) \equiv \xi^{\text {in }}\left(\alpha_{e} \mathbf{Q}-\alpha_{h} \mathbf{Q}^{\prime},-\alpha_{e} \mathbf{Q}-\alpha_{h} \mathbf{Q}^{\prime}\right) .
$$

The function $\xi^{\text {in }}(\mathbf{u}, \mathbf{v})$, which does not explicitly depend on the mass ratio, is precisely given by

$$
\begin{gathered}
\xi^{\text {in }}(\mathbf{u}, \mathbf{v})=\sum_{\mathbf{q} \neq \mathbf{0}, \mathbf{k}, \eta= \pm 1} V_{\mathbf{q}} \\
\left\langle\nu_{0} \mid \mathbf{k}+\frac{\mathbf{v}+\mathbf{q}}{2}\right\rangle\left\langle\nu_{0} \mid \mathbf{k}-\frac{\mathbf{v}+\mathbf{q}}{2}\right\rangle\left\langle\mathbf{k}+\frac{\mathbf{u}+\eta \mathbf{q}}{2} \mid \nu_{0}\right\rangle \\
\times\left[\left\langle\mathbf{k}-\frac{\mathbf{u}+\eta \mathbf{q}}{2} \mid \nu_{0}\right\rangle-\left\langle\mathbf{k}-\frac{\mathbf{u}-\eta \mathbf{q}}{2} \mid \nu_{0}\right\rangle\right],
\end{gathered}
$$

so that $\xi^{\text {in }}(\mathbf{u}, \mathbf{v})=\xi^{\text {in }}(-\mathbf{u}, \mathbf{v})=\xi^{\text {in }}(-\mathbf{u},-\mathbf{v})$.

\section{Effective scattering}

Since a $\left(\mathbf{P}^{\prime} \leftrightarrow-\mathbf{P}^{\prime}\right)$ exchange in Eq.(5) amounts to change $\mathbf{Q}$ into $\mathbf{Q}^{\prime}$, the effective scattering for excitons going from states $\left(\nu_{0}, \mathbf{P}\right),\left(\nu_{0},-\mathbf{P}\right)$ to states $\left(\nu_{0}, \mathbf{P}^{\prime}\right),\left(\nu_{0},-\mathbf{P}^{\prime}\right)$, ends by reading as

$$
\xi^{\mathrm{eff}}\left(\begin{array}{rr}
-\mathbf{P}^{\prime} & -\mathbf{P} \\
\mathbf{P}^{\prime} & \mathbf{P}
\end{array}\right) \equiv \xi_{\alpha_{e}}^{\mathrm{eff}}\left(\mathbf{Q}, \mathbf{Q}^{\prime}\right)
$$

$$
\begin{array}{r}
=\left[\xi_{\alpha_{e}}^{\operatorname{dir}}(Q)-\xi^{\mathrm{in}}\left(\alpha_{e} \mathbf{Q}-\alpha_{h} \mathbf{Q}^{\prime},-\alpha_{e} \mathbf{Q}-\alpha_{h} \mathbf{Q}^{\prime}\right)\right] \\
+\left[\mathbf{Q} \leftrightarrow \mathbf{Q}^{\prime}\right],
\end{array}
$$

where $\mathbf{Q}$ and $\mathbf{Q}^{\prime}$ are the momentum transfers defined in Eq.(5) in terms of the exciton initial and final momenta $\left(\mathbf{P}, \mathbf{P}^{\prime}\right)$.

We have seen that, for equal electron and hole masses, i.e., for $\alpha_{e}=1 / 2$, the direct Coulomb scattering cancels. Due to the symmetry properties of $\xi^{\text {in }}(\mathbf{u}, \mathbf{v})$, the effective exciton-exciton scattering then reduces to one term only

$$
\begin{aligned}
\xi_{1 / 2}^{\mathrm{eff}}\left(\mathbf{Q}, \mathbf{Q}^{\prime}\right) & =-2 \xi^{\mathrm{in}}\left(\left(\mathbf{Q}-\mathbf{Q}^{\prime}\right) / 2,\left(-\mathbf{Q}-\mathbf{Q}^{\prime}\right) / 2\right) \\
& =-2 \xi^{\mathrm{in}}\left(\mathbf{P}^{\prime}, \mathbf{P}\right) .
\end{aligned}
$$

In the other limit, i.e., when the hole mass is infinite, $\alpha_{e}$ is equal to zero. Due to the symmetry properties of $\xi^{\text {in }}(\mathbf{u}, \mathbf{v})$, this effective scattering is then given by

$$
\begin{aligned}
\xi_{0}^{\mathrm{eff}}\left(\mathbf{Q}, \mathbf{Q}^{\prime}\right) & =\left[\xi_{0}^{\operatorname{dir}}(Q)-\xi^{\mathrm{in}}\left(-\mathbf{Q}^{\prime},-\mathbf{Q}^{\prime}\right)\right]+\left[\mathbf{Q} \leftrightarrow \mathbf{Q}^{\prime}\right] \\
& =\left[\xi_{0}^{\operatorname{dir}}(Q)-\xi^{\text {in }}(\mathbf{Q}, \mathbf{Q})\right]+\left[\mathbf{Q} \leftrightarrow \mathbf{Q}^{\prime}\right]
\end{aligned}
$$

\section{POSSIBLE CANCELLATION OF THE EFFECTIVE EXCITON-EXCITON SCATTERING}

\section{A. Previous work}

In a previous work ${ }^{36}$, we already calculated the "in" exchange Coulomb scattering in the particular case of initial excitons having equal momenta in the laboratory frame. This configuration corresponds to $\mathbf{P}=\mathbf{0}$, i.e., $\mathbf{Q}^{\prime}=-\mathbf{Q}$. According to Eq.(24), this means that we already calculated

$$
\xi^{\text {in }}\left(\mathbf{Q},\left[\alpha_{h}-\alpha_{e}\right] \mathbf{Q}\right)=\xi_{\alpha_{e}}^{\text {in }}(Q) .
$$

By contrast with the direct scattering which stays positive, this "in" exchange scattering is negative for small $Q$ but turns positive when $Q$ gets large. More precisely, for zero momentum transfer, $\mathbf{Q}=0$, i.e., when the two ground state excitons $\left(\nu_{0}, \mathbf{0}\right)$ stay in the same $\left(\nu_{0}, \mathbf{0}\right)$ state, $\xi_{\alpha_{e}}^{\text {in }}(0)$ in $2 \mathrm{D}$ is equal to ${ }^{38}-\left(4 \pi-315 \pi^{3} / 1024\right) \simeq$ -3.0 whatever the carrier masses are, while $\xi_{\alpha_{e}}^{\text {in }}(Q)$ turns positive for a momentum transfer $Q_{\alpha_{e}}^{(0)}$ which slightly varies with $\alpha_{e}$,

$$
\xi_{\alpha_{e}}^{\text {in }}(Q)=0, \text { for } Q=Q_{\alpha_{e}}^{(0)} .
$$

For infinite hole mass, this momentum transfer is equal to $Q_{0}^{(0)} \simeq 2.7$.

In this previous work, we also calculated $\xi_{\alpha_{e}}^{\mathrm{eff}}\left(\mathbf{Q}, \mathbf{Q}^{\prime}\right)$ for $\mathbf{P}=\mathbf{0}$. Since $\mathbf{P}=\mathbf{0}$ corresponds to $\mathbf{Q}^{\prime}=-\mathbf{Q}$, we thus also know

$$
\xi_{\alpha_{e}}^{\mathrm{eff}}(\mathbf{Q},-\mathbf{Q}) \equiv \xi_{\alpha_{e}}^{\mathrm{eff}}(Q)
$$

This effective scattering was found to cancel for a momentum transfer which slightly depends on mass ratio,

$$
\xi_{\alpha_{e}}^{\mathrm{eff}}(Q)=0 \text { for } Q=Q_{\alpha_{e}}^{*} .
$$


$Q_{\alpha_{e}}^{*}$ varies from $Q_{1 / 2}^{*} \simeq 3.1$ to $Q_{0}^{*} \simeq 3.9$ when the hole mass increases, the effective scattering $\xi_{\alpha_{e}}^{\mathrm{eff}}(Q)$ staying very close to zero for $Q$ larger than $Q_{\alpha_{e}}^{*}$. However, since energy conserving scattering when $\mathbf{P}=\mathbf{0}$ imposes $\mathbf{P}^{\prime}=$ 0, i.e., $Q=0$, such a momentum transfer $Q_{\alpha_{e}}^{*}$ for cancellation of $\xi^{\text {eff }}$ is of no physical relevance because it corresponds to process in which energy is not conserved.

When $P=0$, the scattered state having the same energy as the initial state corresponds to $P^{\prime}=P$ : it thus reduces to the initial state, which makes the $P=0$ initial configuration not so much of interest. In order to consider physically relevant energy conserving configurations, we must extend our previous calculations to finite initial momentum difference.

Since the direct Coulomb scattering is analytically known, this means that we have to numerically calculate the "in" exchange Coulomb scattering for arbitrary initial momenta. From it, we will then determine, for various scattered angles $\theta$ and mass ratios $\alpha_{e}$, the value of the initial momentum difference for which the effective scattering defined in Eq.(26) cancels when $P=P^{\prime}$, i.e., when energy is conserved. Let us call $P_{\alpha_{e}}^{(0)}(\theta)$ the $P$ value for which such cancellation occurs,

$$
\xi_{\alpha_{e}}^{\mathrm{eff}}\left(\mathbf{Q}, \mathbf{Q}^{\prime}\right)=0 \text { for } P=P^{\prime}=P_{\alpha_{e}}^{(0)}(\theta)
$$

The resulting $\theta$ dependence of this half initial momentum difference is given in Figs 9 and 11 for infinite hole mass and equal electron and hole masses. Let us now derive these results more in details.

\section{B. Infinite hole mass}

Actually, our previous work ${ }^{36}$ in which we only considered $P=0$, is enough to get the effective scattering for a general $\left(\mathbf{P}, \mathbf{P}^{\prime}\right)$ configuration when the hole mass is infinite, i.e., when $\alpha_{e}=0$. Indeed, the "in" exchange scattering in Eq. (24) then reduces to $\xi^{\text {in }}\left(-\mathbf{Q}^{\prime},-\mathbf{Q}^{\prime}\right)$ which is nothing but $\xi_{0}^{\text {in }}\left(Q^{\prime}\right)$, according to Eq.(29). Consequently, the effective scattering, given in Eq.(28) reads as

$$
\begin{aligned}
\xi_{0}^{\mathrm{eff}}\left(\mathbf{Q}, \mathbf{Q}^{\prime}\right) & =\left[\xi_{0}^{\mathrm{dir}}(Q)-\xi_{0}^{\mathrm{in}}(Q)\right]+\left[Q \leftrightarrow Q^{\prime}\right] \\
& =\frac{1}{2}\left[\xi_{0}^{\mathrm{eff}}(Q)+\xi_{0}^{\mathrm{eff}}\left(Q^{\prime}\right)\right]
\end{aligned}
$$

Due to Eq.(32), this effective scattering obviously cancels for $Q=Q^{\prime}=Q_{0}^{*}$. It also cancels for configurations having different momentum transfers, $Q$ and $Q^{\prime}$ then being on both sides of $Q_{0}^{*}$. If we now restrict to processes in which energy is conserved, $Q^{2}+Q^{\prime 2}=4 P^{2}$, this cancellation occurs when half the initial momentum difference $P$ is larger than a threshold value $P_{0}^{*}$ which precisely corresponds to equal momentum transfers $Q=Q^{\prime}=Q_{0}^{*}$, so that this initial momentum threshold corresponds to

$$
P_{0}^{*}=Q_{0}^{*} / \sqrt{2} \simeq 2.77
$$

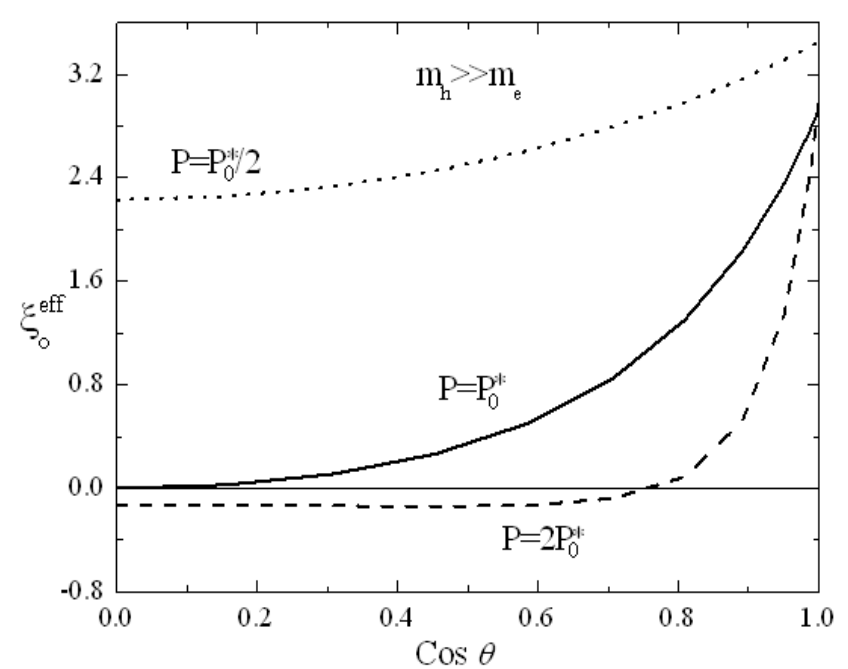

FIG. 7: Effective scatterings $\xi_{\alpha_{e}=0}^{\text {eff }}$ in the case of infinite hole mass, $m_{e} / m_{h}=0$, for three different values of half the initial momentum difference $P$, namely, $P_{0}^{*} / 2, P_{0}^{*}, 2 P_{0}^{*}$, where $P_{0}^{*}$ is the threshold value of $\mathrm{P}$ above which the effective excitonexciton scattering can cancel.

Since $\mathbf{P} . \mathbf{P}^{\prime}=0$ for $Q=Q^{\prime}$, cancellation at threshold occurs for scattering in the perpendicular direction $(\cos \theta=0)$. For $P$ larger than this threshold value $P_{0}^{*}$, the angle $\theta$ between $\mathbf{P}$ and $\mathbf{P}^{\prime}$ when cancellation occurs, decreases, the effective scattering staying however small for all configurations. This means that, for $P \gg P_{0}^{*}$, sizeable scatterings exist in the forward direction only $(\cos \theta \simeq \pm 1)$, i.e., $\theta \simeq(0$ or $\pi)$. This behavior is shown in Fig.7, which gives the effective scatterings for three different values of $P$ taken below, at and above threshold, namely, $P=P_{0}^{*} / 2, P_{0}^{*}$ and $2 P_{0}^{*}$. We in particular see that, for $P=2 P_{0}^{*}$, cancellation occurs for $\cos \theta \simeq 0.75$.

Using Eq.(34), it is possible to calculate the effective scattering as a function of half the momentum difference $P$ and the angle $\theta$ between scattered momenta. In Fig.8, the effective scattering landscape for energy conserving configurations is shown for a large range of $P$ values. The heavy line corresponds to zero effective scattering. Sections with planes where $P$ is constant give curves similar to the ones of Fig.7. Since the direct Coulomb scattering $\xi^{\text {dir }}$ is always positive [see Eq.(23)], Fig.8 shows how compensation between direct and exchange Coulomb processes evolves to produce cancellation. The $\xi_{0}^{\text {eff }}=0$ heavy line separates the effective scattering surface into two regions; when $\xi_{0}^{\text {eff }}<0$, the exchange term dominates over the direct one as in the small momentum limit.

In order to better characterize this cancellation effect, we also show in Fig.9 the curve $P_{0}^{(0)}(\theta)$ [defined in Eq.(33)] where $\xi_{0}^{\text {eff }}$ cancels. Among all possible final states satisfying energy and momentum conservations, this curve selects the forbidden ones at first order in Coulomb process. In agreement with Fig.7, the minimum value of $P_{0}^{(0)}(\theta)$ occurs at threshold $P_{0}^{(0)}(\pi / 2)=P_{0}^{*}$ for 


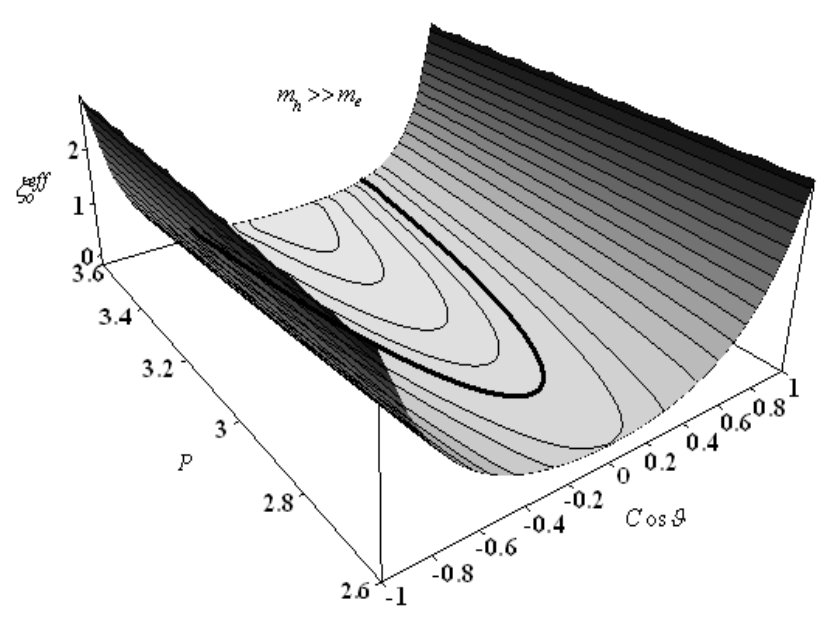

FIG. 8: Effective scatterings $\xi_{\alpha_{e}=0}^{\text {eff }}$ for energy conserving processes as a function of half the initial momentum difference $\mathrm{P}$ and the angle $\theta$ between initial and scattered momenta when the hole mass is infinite. The full lines correspond to constant $\xi_{\alpha_{e}=0}^{\mathrm{eff}}$, the heavy one corresponding to $\xi_{\alpha_{e}=0}^{\text {eff }}=0$.

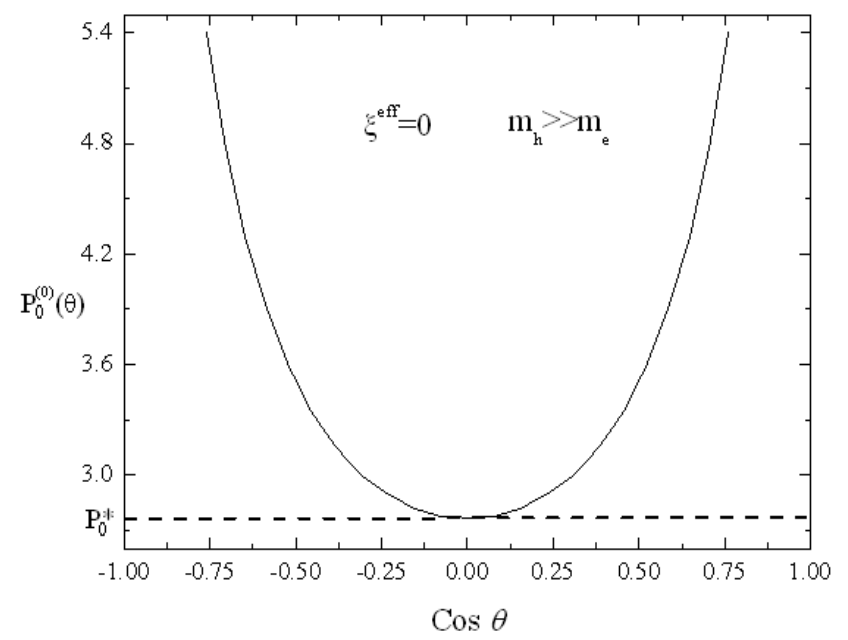

FIG. 9: Initial half exciton momentum difference $P_{\alpha_{e}=0}^{(0)}(\theta)$ for effective scattering cancellation as a function of the angle between initial and scattered momenta when the hole mass is infinite. The dashed line corresponds to the treshold value $P_{0}^{*} \simeq 2.77$ above which cancellation can occur [see Eq.(35)].

$\cos \theta=0$, while for $2 P_{0}^{*}$ it occurs for $\cos \theta \simeq \pm 0.75$.

\section{Equal electron and hole masses}

We now turn to the other limit, i.e., equal electron and hole masses. As seen from Eq.(27), the effective scattering in the forward direction, $\mathbf{P}=\mathbf{P}^{\prime}$, reduces to $-2 \xi^{\text {in }}(\mathbf{P}, \mathbf{P})$ which is nothing but $-2 \xi_{0}^{\text {in }}(P)$, due to Eq.(29). This effective scattering is thus found to cancel for $P$ equal to $Q_{0}^{(0)} \simeq 2.7$ [see Eq.(30)], so that the momentum $P_{1 / 2}^{(0)}(\theta=0)$ for cancellation of the effective

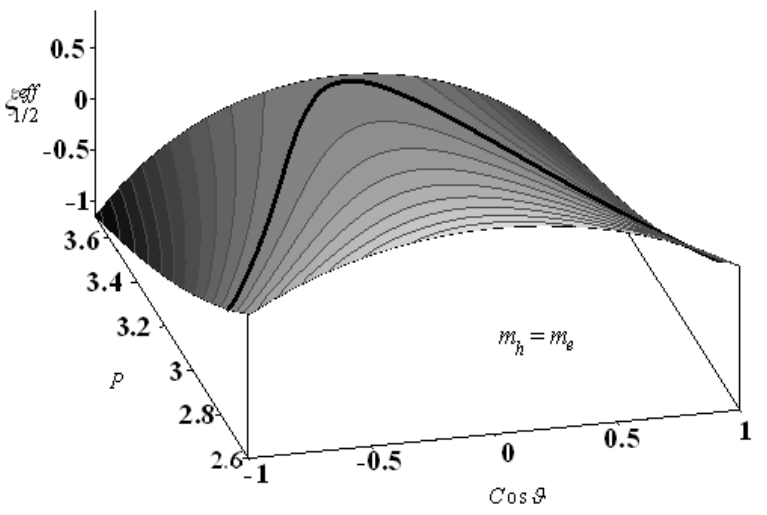

FIG. 10: Effective scatterings $\xi_{\alpha_{e}=1 / 2}^{\text {eff }}$ for energy conserving processes as a function of half the initial momentum difference $\mathrm{P}$ and the angle $\theta$ between initial and scattered momenta when the electron and hole masses are equal. The full lines correspond to constant $\xi_{\alpha_{e}=1 / 2}^{\text {eff }}$, the heavy one corresponding to $\xi_{\alpha_{e}=1 / 2}^{\text {eff }}=0$.

scattering, defined in Eq.(33), is equal to $Q_{0}^{(0)}$.

Through a numerical calculation of the scattering $\xi^{\text {in }}\left(\mathbf{P}^{\prime}, \mathbf{P}\right)$ given in Eq. $(25)$, when energy is conserved, i.e., for $P=P^{\prime}$, we can get the effective scattering $\xi_{1 / 2}^{\text {eff }}$ as a function of half the momentum difference $P$ and scattering angle $\theta$ and determine where it cancels when energy is conserved. By comparing Figs.(8) and (10), we see that the behaviours of $\xi_{\alpha_{e}=0}^{\mathrm{eff}}$ and $\xi_{\alpha_{e}=1 / 2}^{\mathrm{eff}}$ for energy conserving configurations are quite different. Most strikingly, the curve $P_{1 / 2}^{(0)}(\theta)$ for cancellation, shown more in details in Fig.11, has a curvature opposite to the one for infinite hole mass, shown in Fig.9: scattering in the perpendicular direction, $\mathbf{P}^{\prime} . \mathbf{P}=0$, is found to cancel for a $P$ value $P_{1 / 2}^{(0)}(\theta=\pi / 2) \simeq 3.66$ which is larger than the value in the forward direction $P_{1 / 2}^{(0)}(\theta=0) \simeq 2.7$. By contrast, the minimum value of $P_{\alpha_{e}=0}^{(0)}(\theta)$, when the hole mass is infinite, is reached for $\theta=0$.

Such different behaviors of $P_{0}^{(0)}(\theta)$ and $P_{1 / 2}^{(0)}(\theta)$ can look quite strange at first, because, from the behaviors of these two extreme mass ratios, $m_{e} / m_{h}=0$ and $m_{e}=m_{h}$, we expect, by continuity, to go through a value of $\alpha_{e}=m_{e} /\left(m_{e}+m_{h}\right)$ for which $P_{\alpha_{e}}^{(0)}(\theta)$ would stay constant and equal to zero when changing the angle $\theta$ between the initial and final state exciton momenta: for half initial momentum difference equal to this constant value, the effective scattering would then stay equal to zero in all directions.

Let us now determine the value of the electron-to-hole mass ratio which separates these two different regimes of curvature, in order to characterize more in details the magic initial configuration having a zero effective scattering in all directions. 


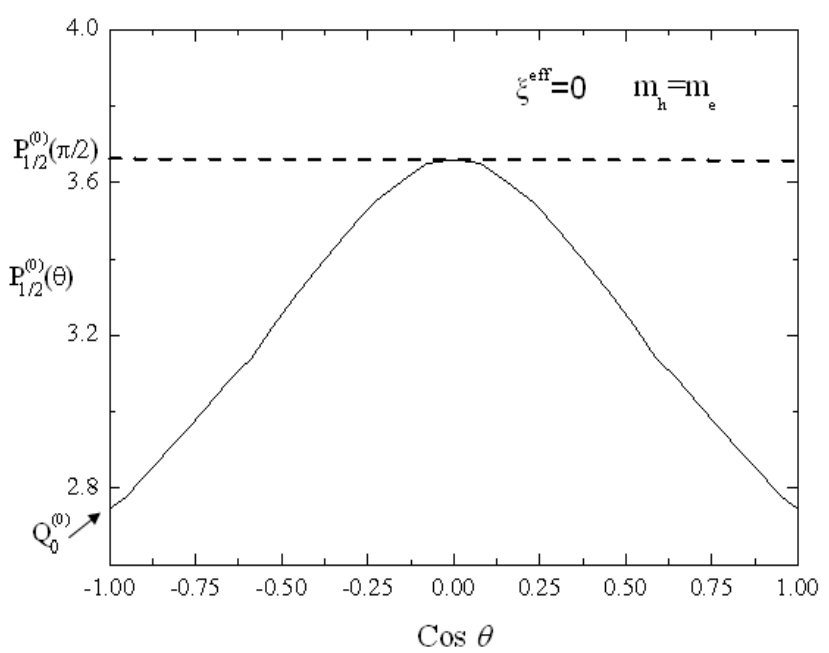

FIG. 11: Initial half exciton momentum difference $P_{\alpha_{e}=1 / 2}^{(0)}(\theta)$ for effective scattering cancellation as a function of the angle between initial and scattered momenta for equal electron and hole masses.

\section{Arbitrary mass ratio}

We start from the general form of $\xi^{\mathrm{eff}}\left(\begin{array}{cc}-\mathbf{P}^{\prime} & -\mathbf{P} \\ \mathbf{P}^{\prime} & \mathbf{P}\end{array}\right)$ given in Eq.(26) which, together with Eqs.(23) and (25), gives the effective scattering as a function of the mass ratio $\alpha_{e}$ and the momentum transfers $\left(\mathbf{Q}, \mathbf{Q}^{\prime}\right)$, these momentum transfers being related to the exciton initial and final momenta $\left(\mathbf{P}, \mathbf{P}^{\prime}\right)$ through Eq.(5). We again consider energyconserving processes, i.e., processes such that $P=P^{\prime}$. The effective scattering then depends on the initial half momentum difference $P$, the angle $\theta$ between $\mathbf{P}$ and $\mathbf{P}^{\prime}$ and the mass ratio $\alpha_{e}$. Let us call it $\xi_{\alpha_{e}}^{\mathrm{eff}}(P, \theta)$.

In order to better see if an initial state with a zero effective scattering in all scattered directions can exist, we first look for values of the initial half momentum difference $\bar{P}$, and mass ratio $\bar{\alpha}_{e}$, for which the effective scattering cancels in the forward and perpendicular directions. This corresponds to look for $\bar{P}$ and $\bar{\alpha}_{e}$ such that

$$
\xi_{\bar{\alpha}_{e}}^{\mathrm{eff}}(\bar{P}, 0)=\xi_{\bar{\alpha}_{e}}^{\mathrm{eff}}(\bar{P}, \pi / 2)=0 .
$$

We find that this happens for $\bar{P} \simeq 3.3$ and $\bar{\alpha}_{e} \simeq 0.32$, which corresponds to a hole mass value $m_{h}$ of the order of $2.08 m_{e}$. Figure 12 shows the half momentum difference $P_{\bar{\alpha}_{e}}^{(0)}(\theta)$ for effective scattering cancellation when the mass ratio is equal to $\bar{\alpha}_{e}$. We see that $P_{\bar{\alpha}_{e}}^{(0)}(\theta)$ is indeed equal to $\bar{P}$ for $\theta=0$ and $\theta=\pi / 2$ but does not stay exactly equal to $\bar{P}$ when changing $\theta$ : this half momentum difference actually shows a very small oscillation, crossing the $P=3.33$ value in four points when $\cos \theta$ varies from -1 to +1 . Although this oscillation is very small, it actually rules out a far more striking behavior, with an effective scattering staying exactly equal to zero for all scattering angles $\theta$.

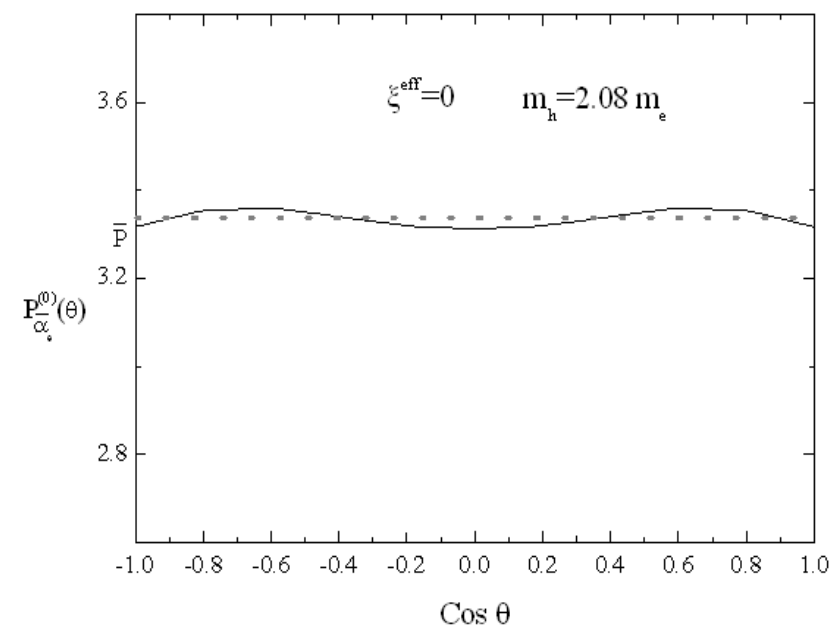

FIG. 12: Initial half exciton momentum difference $P_{\alpha_{e}}^{(0)}(\theta)$ for effective scattering cancellation as a function of the angle between initial and scattered momenta when $m_{h}=2.08 m_{e}$, i.e., when $\alpha_{e}=\bar{\alpha}_{e}=0.32$. The dotted line, $P=3.33$, is to guide the eyes for the weak oscillation of $P_{\bar{\alpha}_{e}}^{(0)}(\theta)$, the value of $P_{\bar{\alpha}_{e}}^{(0)}(\theta)$ being exactly equal to $\bar{P} \simeq 3.3$ for $\cos \theta=(0, \pm 1)$

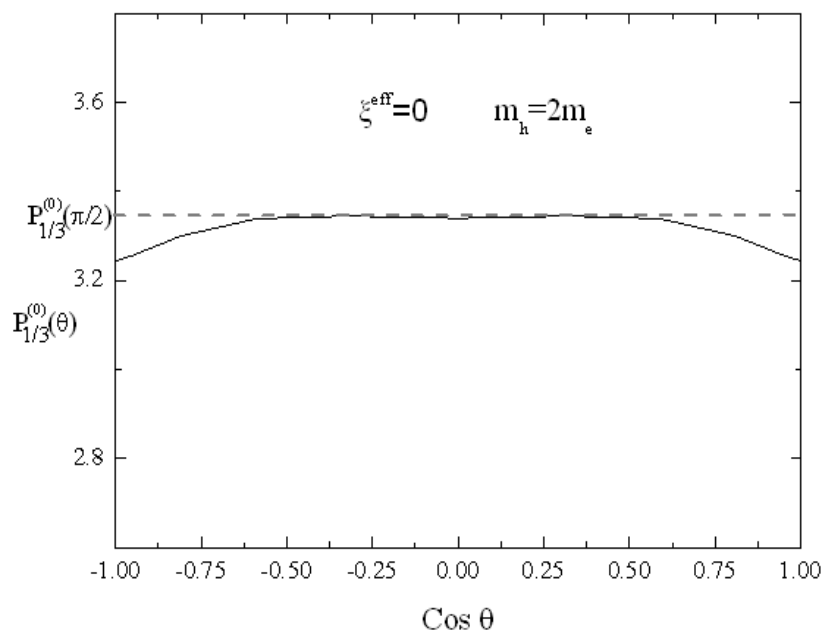

FIG. 13: Half initial exciton momentum difference $P_{\alpha_{e}=1 / 3}^{(0)}(\theta)$ for cancellation of the effective scattering, as a function of the angle between initial and scattered momenta when $m_{h}=$ $2 m_{e}$.

In order to better characterize this fundamental collapse of the effective exciton-exciton scattering, we have performed calculations for values of $\alpha_{e}$ very close to $\bar{\alpha}_{e}$, namely, $m_{h}=(2.08 \mp 0.08) m_{e}$ : the results are shown in Fig.13 and Fig.14 respectively. In both cases, we find that there are very narrow ranges of $P$ values for which scattering cancellation occurs $(\Delta P / P \simeq 0.03)$.

Of particular interest is the case $m_{h}=2 m_{e}$ for which, when the initial half momentum difference $P$ is equal to $P_{1 / 3}^{(0)}(\pi / 2)$ (see Fig.13), the effective scattering stays essentially equal to zero for all scattered directions $\theta$ be- 


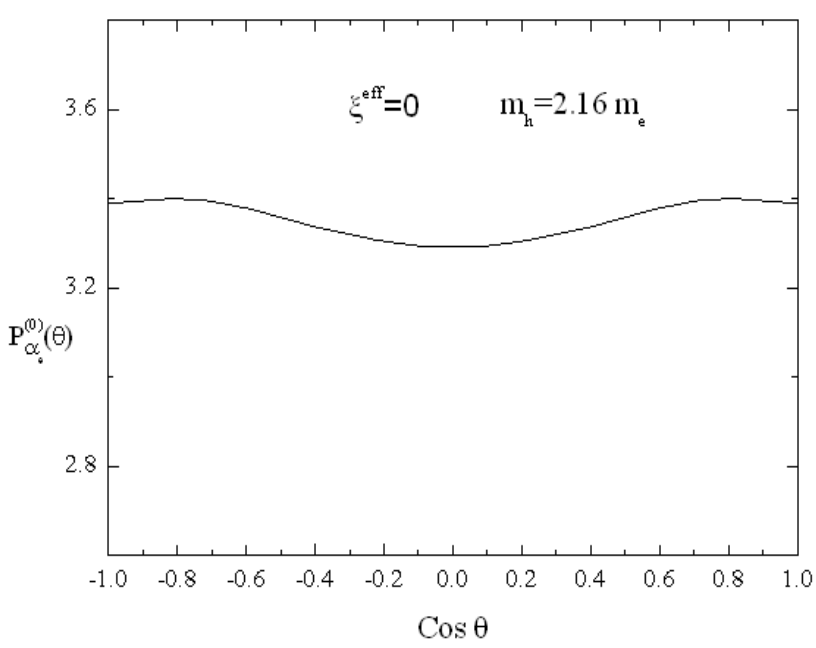

FIG. 14: Half initial exciton momentum difference $P_{\alpha_{e}}^{(0)}(\theta)$ for cancellation of the effective scattering, as a function of the angle between initial and scattered momenta when $m_{h}=$ $2.16 m_{e}$.

tween $\pi / 4$ and $3 \pi / 4$. This rather large range of $\theta$ values in which energy conserving scattering is forbidden, could possibly help to evidence this highly non intuitive fundamental collapse. We see that, for this $P$ value, non-zero scattering exists for $\cos \theta$ very close to \pm 1 , i.e., in the forward direction only.

The $m_{h} / m_{e} \simeq 2.08$ mass ratio, which fundamentally separates two different regimes of curvature for $P_{\alpha_{e}}^{(0)}(\theta)$, can be physically seen as the precise value which separates positronium-like excitons with $m_{e} \simeq m_{h}$ (Fig.8) from hydrogen-like excitons with $m_{e} \ll m_{h}$ (Fig.10).

It is clear that the cancellation of the exciton-exciton effective scattering requires exciton initial momenta far larger than photon momenta, i.e., the momenta of photocreated excitons. As a result, this quite remarkable collapse seems hard to experimentally evidence in a direct way through excitons created by resonant photons. Nevertheless, its existence could have consequences in physical properties related to exciton-exciton scatterings for systems having excitons with high kinetic energy as possibly produced by non-resonant photons.

For example to explain the rise time observed in time resolved luminescence of GaAs/AlGaAs/GaAs(001) 2D multi quantum well, the authors of Ref.[39] speculate that exciton-exciton scattering plays a dominant role with respect to dephasing effects linked to either disorder or acoustic phonons. It is legitimate to then expect strongly different behavior when such dominant mechanism is hampered by the present cancellation effect.

We wish to stress that, although rather large, the half initial exciton momentum difference $P_{\alpha_{e}}^{(0)}$ for which scattering cancels, stays below the exciton ionization threshold, for all mass ratios considered here, as can be seen from Fig.4. Consequently, the asumption of excitons staying in their fundamental ground state, under which these calculations are made, is fully valid.

The present work considers quasi two-dimensional quantum wells $\left(L \ll a_{X}\right)$ with hole-to-electron mass ratio $m_{h} / m_{e}$ close to 2 (Fig.13). This is easily fulfilled in high quality GaAs/AlAs/GaAs(001) with electron mass $m_{e}=0.067$ and hole mass in the parallel direction $m_{h h}^{/ /}=0.110$. The $3 \mathrm{D}$ Bohr radius being $a_{X}=17.5 \mathrm{~nm}$, the $2 \mathrm{D}$ Rydberg energy $4 R_{y d}$ is of the order of $12 \mathrm{meV}$ while the ionization threshold for center-of mass energy is of the order of $7.8 \mathrm{meV}$ (see Fig.4). Moreover, being the difference between the lowest $(n=0)$ and the first excited state $(\mathrm{n}=1)$, for $2 \mathrm{D}$ excitons, $\Delta E \approx 3.55 R_{y d}$ we can check that the exciton kinetic energy is lower than the transition energy $\Delta E$. Moreover, high quality samples are necessary to minimize dephasing induced by interface disorder. A very accurate control of the sample temperature is also needed to get rid of exciton-acoustic phonon interaction. A precise control of the heavy-light hole splitting energy, induced by small difference in the lattice parameters between GaAs and AlAs materials (compressive strain) is also required. Note that this splitting must be added to the quantum confinement energy (for GaAs the z-masses are $\left.m_{h h}^{z}=0.530, m_{l h}^{z}=0.08\right)$.

All this tends to show that, altought the experimental observation of the exciton-exciton scattering cancellation is going to require a rather sophisticated tailoring of the sample as well as non-linear optical experiments designed in an unusual way, the observation of such an unexpected effect which is directly linked to the existence of fermionic components in excitons, does not seem out of reach and seems to us a challenge of physical interest.

\section{CONCLUSION}

Through the composite exciton many-body framework recently proposed by Combescot and coworkers, we here study the effective scattering ruling the time evolution of two excitons at first order in Coulomb interaction (Born approximation) when these two excitons are in the same relative motion state but have different initial momenta. We mainly look for energy conserving configurations in which this effective scattering cancels.

(i) We show that the possible cancellation of this effective scattering is fundamentally due to the exciton composite nature: such a cancellation always occurs for elementary fermions but never occurs for elementary bosons. In the case of composite-boson excitons made of two fermions, cancellation can occur but above a momentum threshold only.

(ii) The effective scattering ruling the time evolution of two excitons shows a strong dependence on the electronto-hole mass ratio. For $m_{h} / m_{e}$ close to 2 , which can be seen as a boundary between hydrogen-like excitons for which $m_{e} \ll m_{h}$ and positronium-like excitons for which $m_{e} \simeq m_{h}$, we find a quite remarkable cancellation of this effective scattering over a large range of scattered directions, $\theta \simeq(\pi / 4,3 \pi / 4)$, but a very narrow range of 
initial exciton momentum difference, $\Delta P / P \simeq 0.03$.

\section{Acknowledgments}

We wish to thank M.A. Dupertuis for a valuable help in the numerical part of this work. One of us (M.C.) wishes to thank the Italian CNR "Istituto dei Sistemi Complessi" for an invitation, through the Short Term Mobility program, during which most of this work was completed.
1 P. G. Savvidis, J. J. Baumberg, R. M. Stevenson, M. S. Skolnick, D. M. Whittaker, and J. S. Roberts, Phys. Rev. Lett. 84, 1547 (2000).

2 D. N. Krizhanovskii, D. Sanvitto, I. A. Shelykh, M. M. Glazov, G. Malpuech, D. D. Solnyshkov, A. Kavokin, S. Ceccarelli, M. S. Skolnick, and J. S. Roberts, Phys. Rev. B 73, 073303 (2006).

3 A. Baas, J.-Ph. Karr, M. Romanelli, A. Bramati, and E. Giacobino, Phys. Rev. B 70, 161307(R) (2004).

4 N. A. Gippius, I. A. Shelykh, D. D. Solnyshkov, S. S. Gavrilov, Y. G. Rubo, A. V. Kavokin, S. G. Tikhodeev, and G. Malpuech, Phys. Rev. Lett. 98, 236401 (2007).

5 N.Garro, S.P.Kennedy, R.T.Phillips, G.Aichmayer, U.Rossler and L.Vina Phys. Rev. B 67, 121302 (2003)

${ }^{6}$ P. Nozières, Bose-Einstein Condensation, A. Griffin, D.W. Snoke, and S. Stringari, eds., Cambridge University Press, (1995), pp. 16-21.

7 J. Kasprzak, M. Richard, S. Kundermann, A. Baas, P. Jeambrun, J.M.J. Keeling, R. Andre, J.L. Staehli, V. Savona, P.B. Littlewood, B. Deveaud, and L.S. Dang, Nature 443, 409 (2006)

8 R. Balili, V. Hartwell, D. Snoke, L. Pfeiffer, and K. West, Science 316, 1007 (2007)

9 A. Amo et al. Nature 457291 (2009)

10 G. Malpuech, D. D. Solnyshkov, H. Ouerdane, M. M. Glazov, and I. Shelykh, Phys. Rev. Lett. 98, 206402 (2007).

11 S. Utsunomiya, L. Tian, G. Roumpos, C. W. Lai, N. Kumada, T. Fujisawa, M. Kuwata-Gonokami, A. Loffler, S. Hofling, A. Forchel, and Y. Yamamoto, Nat. Phys. 9, 674 (2008).

12 Maxime Richard et al. Int. J. Nanotechnology 7, 668 (2010)

13 S. A. Moskalenko, Sov. Phys. Solid State 4, 199 (1962).

14 L. V. Keldysh and Y. V. Kopaev, Sov. Phys. Solid State 6, 2219 (1965).

15 D.W. Snoke, Science 298, 1368 (2002).

16 Z. Voros, D. W. Snoke, L. Pfeiffer, and K. West Phys. Rev. Lett. 97, 016803 (2006); Phys. Rev. Lett. 103, 016403 (2009)

17 M. Wouters, V. Savona Phys. Rev. B 81, 054508 (2010)

18 C. Ciuti, V. Savona, C. Piermarocchi, and A. Quattropani, Phys. Rev. B 58, 7926 (1998).
19 Jun-ichi Inoue, T. Brandes, and A. Shimizu, Phys. Rev. B 61, 2863 (2000).

20 J. Shumway and D.M. Ceperley, Phys. Rev. B 63, 165209 (2001).

21 C. Schindler and R. Zimmermann, Phys. Rev. B 78, 045313 (2008).

22 M. Combescot, O. Betbeder-Matibet and R. Combescot, Phys. Rev. Lett. 99, 176403 (2007).

23 M. Combescot and O. Betbeder-Matibet, Phys. Rev. B 72, 193105 (2005).

24 M. Combescot and M.-A. Dupertuis, Phys. Rev. B 78, 235303 (2008).

25 M. Combescot and W. Pogosov, Phys. Rev. B 77, 085206 (2008).

26 M. Combescot and W. Pogosov, Eur. Phys. J. B 68, 161 (2009).

27 W. Pogosov and M. Combescot, Eur. Phys. J. B 68, 183 (2009).

28 S. Ben-Tabou De-Leon and B. Laikhtman, Phys. Rev. B 63, 125306 (2001).

29 S. Rombouts, D. Van Neck, K. Peirs and L. Pollet, Mod. Phys. Lett. A 17, 1899 (2002).

30 V. Chernyak and S. Mukamel, J. Opt. Soc. Am. B 13, 1302 (1996).

31 V. Chernyak, S. Yokojima, T. Meier and S. Mukamel, Phys. Rev. B 58, 4496 (1998).

32 S. Mukamel, R. Oszwaldowski and D. Abramavicius, Phys. Rev. B 75, 245305 (2007).

33 M. Combescot, O. Betbeder-Matibet and F. Dubin, Phys. Reports 463, 215 (2008).

34 M. Combescot and O. Betbeder-Matibet, arXiv: $0902.4588 \mathrm{v} 1$.

35 M. Combescot and O. Betbeder-Matibet, Phys. Rev. Lett. 93, 016403 (2004).

36 M. Combescot, O. Betbeder-Matibet and R. Combescot, Phys. Rev. B 75, 174305 (2007).

37 O. Betbeder-Matibet and M. Combescot, Eur. Phys. J. B 27, 505 (2002).

38 M. Combescot, M.-A. Dupertuis and O. Betbeder-Matibet, Europhys. Lett. 79, 17001 (2007).

39 S.Hailin Wang, Jagdeep Shah, T.C.Damen, L.N. Pfeiffer, Phys. Rev. Lett. 74, 3065 (1995) 